\title{
Nelarabine-Induced Myelotoxicity
}

Ajay A. Madhavan, MD, Carrie M. Carr, MD, Hassan Alkhateeb, MD, Nathan P. Staff, MD, and Elie Naddaf, MD Neurology ${ }^{\circledR}$ 2021;96:175-176. doi:10.1212/WNL.0000000000011343

Figure Nelarabine-Induced Myelotoxicity

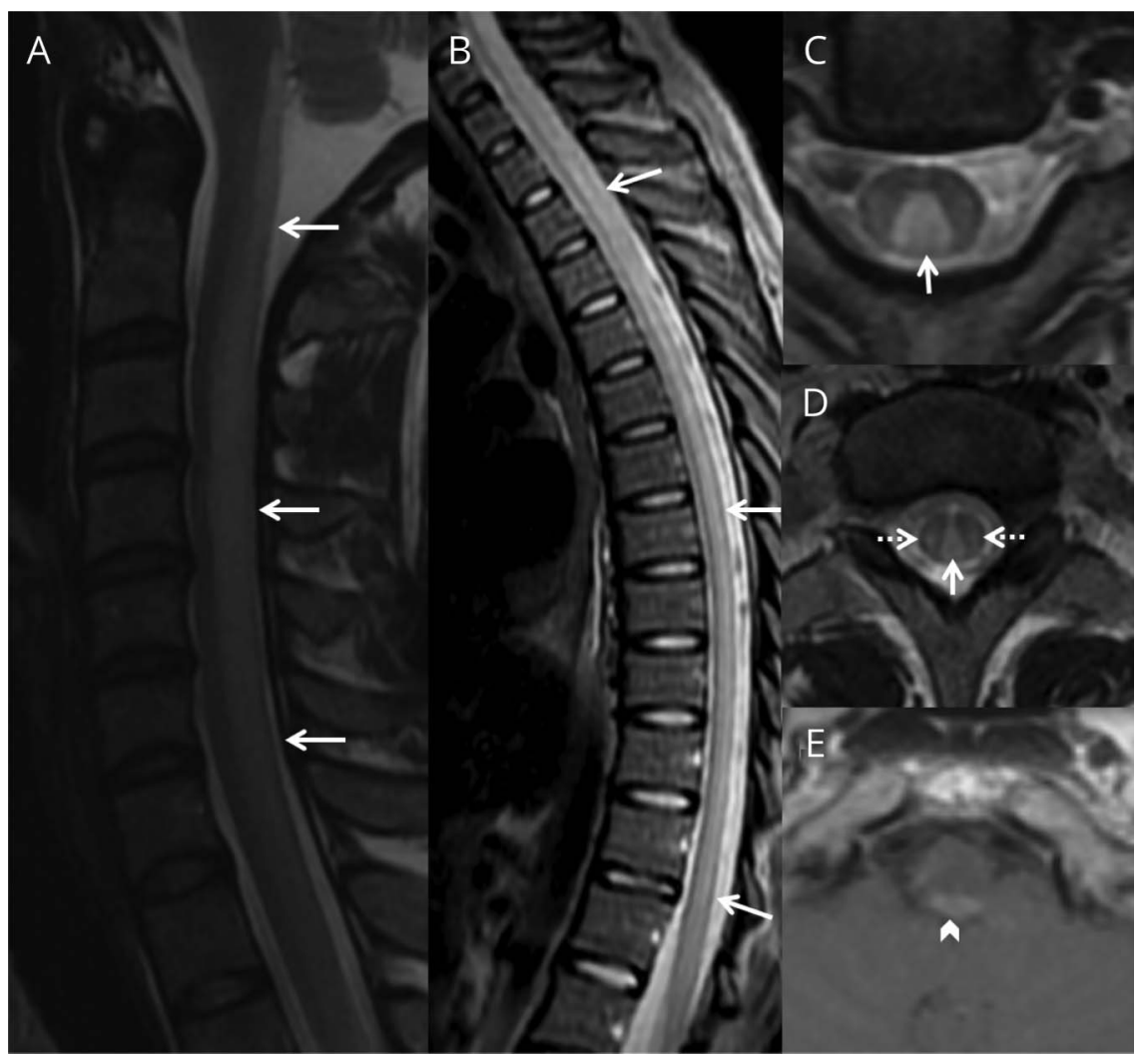

MRI of the cervical ( $A, C$, and $E$ ) and thoracic (B and D) spine. Sagittal T2-weighted (A), sagittal short tau inversion recovery $(B)$, axial T2-weighted (C-D), and axial T1-weighted postcontrast (E) images demonstrate extensive $T 2$ hyperintensity of the dorsal (A-D, arrows) and lateral columns (D, dashed arrows), with dorsal column enhancement at the cervicomedullary junction ( $E$, arrowhead).

A 29-year-old woman with relapsed T-cell acute lymphoblastic leukemia presented with 4 months of progressive ataxia, weakness, and bowel/bladder incontinence. Symptoms started after completing 2 cycles of nelarabine. Examination revealed paraplegia and complete sensory loss from mid-chest down, loss of vibration and joint position in upper limbs, areflexia, and bilateral Babinski signs. EMG showed fibrillation potentials with no activated motor units in lower limbs. Laboratory workup, including paraneoplastic autoantibody panel, CSF analysis, vitamin $\mathrm{B}_{12}$, methylmalonic acid, and copper levels, was unremarkable. Spine MRI findings were consistent with nelarabine-induced subacute

\section{Correspondence}

Elie Naddaf

naddaf.elie@mayo.edu 
combined degeneration, including previously unreported lateral column involvement and cord enhancement (figure). ${ }^{1,2}$ The unremarkable CSF, MRI findings (tractopathy), and subacute course made alternative etiologies such as infection, infiltrative leukemia, ischemia, or cord hemorrhage less likely. The patient was treated conservatively and started to spontaneously improve. She then underwent allogenic stem cell transplantation. Three months later, she regained movement against gravity in all lower extremity muscles. However, she remained severely ataxic, requiring the use of a wheelchair. Repeat EMG showed reinnervation in most lower-limb muscles and less frequent fibrillation potentials.

\section{Study Funding}

No targeted funding reported.

\section{Disclosure}

The authors report no disclosures relevant to the manuscript. Go to Neurology.org/N for full disclosures.
Appendix Authors

\begin{tabular}{|c|c|c|}
\hline Name & Location & Contribution \\
\hline $\begin{array}{l}\text { Ajay A. } \\
\text { Madhavan, MD }\end{array}$ & $\begin{array}{l}\text { Mayo Clinic, } \\
\text { Rochester, MN }\end{array}$ & $\begin{array}{l}\text { Writing and editing the manuscript } \\
\text { and figure }\end{array}$ \\
\hline $\begin{array}{l}\text { Carrie M. Carr, } \\
\text { MD }\end{array}$ & $\begin{array}{l}\text { Mayo Clinic, } \\
\text { Rochester, MN }\end{array}$ & $\begin{array}{l}\text { Study conception, writing and } \\
\text { editing the manuscript and figure }\end{array}$ \\
\hline $\begin{array}{l}\text { Hassan } \\
\text { Alkhateeb, MD }\end{array}$ & $\begin{array}{l}\text { Mayo Clinic, } \\
\text { Rochester, MN }\end{array}$ & Editing the manuscript and figure \\
\hline $\begin{array}{l}\text { Nathan P. } \\
\text { Staff, MD }\end{array}$ & $\begin{array}{l}\text { Mayo Clinic, } \\
\text { Rochester, MN }\end{array}$ & Editing the manuscript and figure \\
\hline $\begin{array}{l}\text { Elie Naddaf, } \\
\text { MD }\end{array}$ & $\begin{array}{l}\text { Mayo Clinic, } \\
\text { Rochester, MN }\end{array}$ & $\begin{array}{l}\text { Study conception, writing and } \\
\text { editing the manuscript and figure }\end{array}$ \\
\hline
\end{tabular}

\section{References}

1. Lalayanni C, Baldoumi E, Papayiannopoulos S, Tziola K, Saloum R, Anagnostopoulos A. Nelarabine-associated reversible Guillain-Barre-like syndrome or myelopathy in an adult patient with primary refractory T-lymphoblastic lymphoma. Curr Probl Cancer 2017;41:138-143.

2. Kawakami M, Taniguchi K, Yoshihara S, et al. Irreversible neurological defects in the lower extremities after haploidentical stem cell transplantation: possible association with nelarabine. Am J Hematol 2013;88:853-857.

\section{Call for Biostatisticians to peer review for Neurology $y^{\circledast}$}

We are seeking qualified experts to review the biostatistical aspects of submitted articles. We welcome those specializing in biostatistics with a background in experimental design and the review of randomized clinical trials, observational studies, genetic studies, and imaging studies in the neurodegenerative diseases.

If interested, please send CV to the Neurology Editorial Office: journal@neurology.org.

\section{Visit the Neurology ${ }^{\circledast}$ Website at Neurology.org/N}

- More article-based content on home pages

- Streamlined menus and navigation

- Enhanced blog sections for specialty areas

- Same experience on desktop, tablet, and mobile devices

- Improved article reading experience; links more evident (pdf, analytics, social media)

- Neurology ${ }^{\circledR}$ Clinical Practice initiative "Practice Current" global surveys will be accessible across sites

f Find Neurology ${ }^{\circledR}$ on Facebook: http://tinyurl.com/neurologyfan

Follow Neurology ${ }^{\circledR}$ on Twitter: https://twitter.com/GreenJournal 


\title{
Neurology
}

\author{
Nelarabine-Induced Myelotoxicity \\ Ajay A. Madhavan, Carrie M. Carr, Hassan Alkhateeb, et al.
}

Neurology 2021;96;175-176 Published Online before print December 11, 2020

DOI 10.1212/WNL.0000000000011343

This information is current as of December 11, 2020

\begin{tabular}{|c|c|}
\hline $\begin{array}{l}\text { Updated Information \& } \\
\text { Services }\end{array}$ & $\begin{array}{l}\text { including high resolution figures, can be found at: } \\
\text { http://n.neurology.org/content/96/4/175.full }\end{array}$ \\
\hline References & $\begin{array}{l}\text { This article cites } 2 \text { articles, } 0 \text { of which you can access for free at: } \\
\text { http://n.neurology.org/content/96/4/175.full\#ref-list-1 }\end{array}$ \\
\hline Citations & $\begin{array}{l}\text { This article has been cited by } 1 \text { HighWire-hosted articles: } \\
\text { http://n.neurology.org/content/96/4/175.full\#\#otherarticles }\end{array}$ \\
\hline Subspecialty Collections & $\begin{array}{l}\text { This article, along with others on similar topics, appears in the } \\
\text { following collection(s): } \\
\text { All Clinical Neurology } \\
\text { http://n.neurology.org/cgi/collection/all_clinical_neurology } \\
\text { All Education } \\
\text { http://n.neurology.org/cgi/collection/all_education } \\
\text { All Spinal Cord } \\
\text { http://n.neurology.org/cgi/collection/all_spinal_cord } \\
\text { MRI } \\
\text { http://n.neurology.org/cgi/collection/mri } \\
\text { Transverse myelitis } \\
\text { http://n.neurology.org/cgi/collection/transverse_myelitis }\end{array}$ \\
\hline Permissions \& Licensing & $\begin{array}{l}\text { Information about reproducing this article in parts (figures,tables) or in } \\
\text { its entirety can be found online at: } \\
\text { http://www.neurology.org/about/about_the_journal\#permissions }\end{array}$ \\
\hline Reprints & $\begin{array}{l}\text { Information about ordering reprints can be found online: } \\
\text { http://n.neurology.org/subscribers/advertise }\end{array}$ \\
\hline
\end{tabular}

Neurology ${ }^{\circledR}$ is the official journal of the American Academy of Neurology. Published continuously since 1951, it is now a weekly with 48 issues per year. Copyright () 2020 American Academy of Neurology. All rights reserved. Print ISSN: 0028-3878. Online ISSN: 1526-632X.

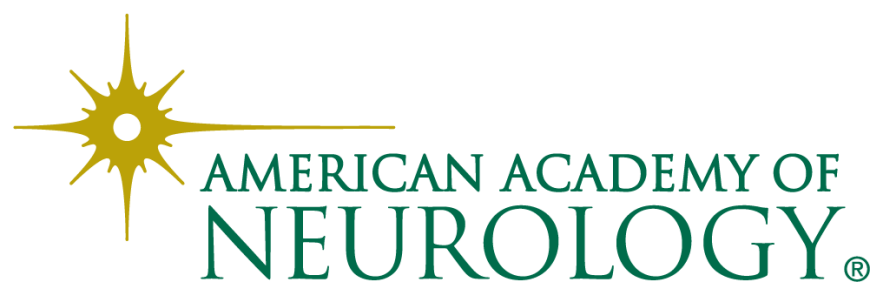

